\title{
Additive-induced Crystallization of Inorganic Perovskite Films for Efficient Solar Cells
}

\author{
Huijie Zhang ${ }^{1}$, Jing Li $^{1}$, Zhen Huang ${ }^{1}$, Wenkang Wang ${ }^{1}$, Jinhua Li, \\ Duofa Wang ${ }^{1,2,3}{ }^{*}$, and Tianjin Zhang ${ }^{1,2,3 *}$ \\ ${ }^{1}$ Hubei Collaborat ive Innovation Center for Advanced Organic Chemical Materials, Hubei University, \\ Wuhan 430062, People's Republic of China \\ ${ }^{2}$ Key Laboratory for the Green Preparation and Application of Functional Materials, Ministry of \\ Education, Hubei University, Wuhan 430062, People's Republic of China \\ ${ }^{3}$ School of Material Science and Engineering, Hubei University, Wuhan 430062, People's Republic of \\ China \\ *E-mail: duofawang@hotmail.com, zhangtj@hubu.edu.cn
}

doi: $10.20964 / 2018.05 .15$

Received: 15 November 2017 / Accepted: 25 February 2018 / Published: 10 April 2018

In recent years, hybrid perovskite solar cells (PSCs) have received extensive attention because of their high power conversion efficiency (PCE), yet their thermal stability is still not ideal. Meanwhile, allinorganic PSCs have excellent thermal stability, but their device efficiency is relatively low. Here we report the use of non-toxic additive-induced crystallization to improve film quality to enhance the efficiency of all-inorganic PSCs. High-quality perovskite films with full surface coverage and uniform grain structure were prepared. Kinetic analysis of the crystallization process showed that the non-toxic additive increased the supersaturation of the perovskite precursor and induced rapid nucleation and crystallization. The optical band gap of the perovskite films was tuned by adjusting iodide content. $\mathrm{CsPbBrI}_{2}$ with an appropriate bandgap was used as the light-absorbing material in PSCs that displayed high thermal stability. By optimizing the preparation process, an all-inorganic PSC with a planar structure delivered a PCE of 5.6\%. The entire device fabrication process was performed in the ambient environment without humidity control. Thus, we developed a simple, controllable, and versatile approach to produce high-quality inorganic perovskite films for use in high-performance photovoltaic devices.

Keywords: all-inorganic; perovskite solar cells; nucleation; crystallization

\section{$\underline{\text { FULL TEXT }}$}


(C) 2018 The Authors. Published by ESG (www.electrochemsci.org). This article is an open access article distributed under the terms and conditions of the Creative Commons Attribution license (http://creativecommons.org/licenses/by/4.0/). 